\title{
Antisense telomerase RNA inhibits the growth of human glioma cells in vitro and in vivo
}

\author{
YONGPING YOU ${ }^{1,2}$, PEIYU PU ${ }^{1}$, QIANG HUANG ${ }^{1}$, ZHIBO XIA $^{1}$, CHUNYAN WANG $^{1}$, \\ GUANGXIU WANG ${ }^{1}$, CHUNZHAO YU ${ }^{2}$, JING JIE YU ${ }^{3}$, EDDIE REED ${ }^{3}$ and QINGDI Q. LI $^{3}$ \\ ${ }^{1}$ Department of Neurosurgery, Tianjin Medical University General Hospital, Tianjin 300052; \\ ${ }^{2}$ Department of Neurosurgery, First Hospital of Nanjing Medical University, Nanjing 210029, P.R. China; \\ ${ }^{3}$ Mary Babb Randolph Cancer Center, and Department of Microbiology, Immunology and Cell Biology, \\ West Virginia University Health Sciences Center, Morgantown, WV 26506, USA
}

Received December 19, 2005; Accepted February 6, 2006

\begin{abstract}
Telomerase is implicated in the development of cellular immortality and oncogenesis. It has been shown that telomerase activity is considerably higher in the tissue of many different cancers than in normal tissue, and that the inhibition or downregulation of telomerase activity can prevent the malignant proliferation of tumor cells. Antisense oligonucleotides have been widely used in suppressing the expression of genes and, therefore, in the present research, we evaluated the effect of antisense human telomerase RNA (hTR) on glioma cell growth in vitro and in vivo. We showed that antisense hTR cDNA significantly inhibited TJ905 human glioma cell proliferation in vitro and tumor growth in vivo, as determined by MTT assay and by measuring the volume of glioma in nude mice. Consistent with these results, we found that telomerase activity and the mRNA levels of hTR and hTERT (human telomerase reverse transcriptase) expression were markedly decreased in tumor cells treated with antisense hTR cDNA, as assessed by TRAP (telomeric repeat amplification protocol) assay and RT-PCR (reverse transcription-polymerase chain reaction) analysis. Our study conclusively demonstrates that antisense hTR effectively inhibits the growth of human glioma cells in vitro and in vivo and, thus, may be potentially used for gene therapy of malignant gliomas and other cancers.
\end{abstract}

Correspondence to: Professor Peiyu Pu, Department of Neurosurgery, Tianjin Medical University, 154 Anshan Road, Tianjin 300052, P.R. China

E-mail: ppyu@public.tpt.tj.cn

Professor Qingdi Q. Li, 216 Watkins Pond Boulevard, Rockville, MD 20850-5622, USA

E-mail: quentinli2004@yahoo.com

Key words: antisense human telomerase RNA, human telomerase reverse transcriptase, hTERT, gliomas, cell proliferation, telomerase activity, gene therapy

\section{Introduction}

Gliomas are the most common type of primary intracranial tumors and constitute $40-50 \%$ of brain tumors. Patients suffering from malignant gliomas in the brain have a life-span of only 9-12 months after diagnosis. Today the conventional treatment available for gliomas includes surgery, radiation therapy, and chemotherapy. Although a great deal of effort has been directed toward the therapy of gliomas, the prognosis of patients has not greatly improved. The main reason for the poor prognosis is due to the fact that the majority of proliferating glioma cells grow in the rim of tumors or "normal brain tissue', and conventional therapies can not selectively kill these carcinoma cells. Therefore, it is a top priority to develop new therapeutic strategies for malignant gliomas.

With the development and advances of molecular biology and its applications in the study of tumorigenesis, it has been demonstrated that the development of malignant tumors, including gliomas, is due to the activation of proto-oncogenes and inactivation of tumor suppressor genes leading to loss of control of cell proliferation and apoptosis, and deficit of normal limited life-span of cells, resulting in cellular immortality and tumorigenesis. Recently, investigators discovered that cellular immortality was caused by activated telomerase. The role of telomerase is to maintain telomeric length and promote cell proliferation potential. The activation of telomerase is considered to be a critical event in cellular immortality.

Evidence is accumulating that telomerase is able to use its RNA component as a template for synthesizing telomeric repeats to lengthen the telomere and cause cellular immortality, which is a critical event in tumor formation. Telomerase is a ribonucleoprotein enzyme complex which elongates telomeric DNA $(\text { TTAGGG) })_{n}$ expressed in $>90 \%$ of advanced cancers but not in most normal proliferating cells (1-5), and is believed to be an important step in the development of cellular immortality and oncogenesis. The most recent and significant advances in this area show that the inhibition or downregulation of telomerase activity would inhibit the malignant proliferation of tumor cells, and restore mortality to tumor cells that express this enzyme (6-8). 
In the last decade, many gene therapy strategies have been developed and used to treat malignant gliomas in animal models, but their therapeutic effects have not been satisfactory. One reason for the poor outcome is the selection of therapeutic genes. Our previous studies and those of others have demonstrated that human telomerase RNA (hTR) and telomerase activity may play an important role in the development and progression of malignant gliomas and may be potentially used in the clinic as a novel target of gene therapy for malignant gliomas. A number of approaches have been developed to achieve telomerase inhibition in tumor cells, based on the use of antisense oligonucleotides, peptide nucleic acids (PNA), or hammerhead ribozymes directed against the core enzyme components. However, how telomerase inhibition affects the proliferation capacity of cancer cells has not yet been clarified.

The present study was designed to investigate the effects of antisense human telomerase RNA (hTR) on glioma cell growth. We constructed an expression vector consisting of hTR antisense cDNA against the template of hTR, and found that incubation of TJ905 human glioma cells with antisense hTR cDNA markedly reduced the expression of hTR and telomerase activity and significantly inhibited the growth of TJ905 glioma cells in vitro and in vivo.

\section{Materials and methods}

Expression vector preparations. The plasmid, pcDNA3.1anti-hTR, bearing the 125-bp cDNA antisense aimed directly at hTR (including the template region) and the plasmid, pcDNA3.1-sense-hTR, bearing the 125-bp cDNA encoding the hTR were gifts from Dr Xiuwen Liu (NIH, Bethesda, MD, USA). The plasmids, pcDNA3.1-anti-hTR and pcDNA3.1sense-hTR, used for transfection were isolated from $E$. coli strain DH5 and purified using the Wizard Plus SV Minipreps kit (Promega, Madison, WI, USA). The DNA concentration and purity were determined using an ultraviolet scanning spectrophotometer. The isolated hTR antisense cDNA was confirmed by $1 \%$ agarose gel electrophoresis after digestion with the restriction enzymes, BamHI and EcoRI (Fermentas).

Cell culture and transfections. The human glioma cell line, TJ905 (its telomerase activity is very high as detected by a modified TRAP assay), from our laboratory was grown in Dulbecco's modified Eagle's medium (DMEM), supplemented with $10 \%$ fetal calf serum and $1 \%$ penicillin-streptomycin, and was maintained at $37^{\circ} \mathrm{C}$ with $5 \% \mathrm{CO}_{2}$. One or two days before transfection, cells were seeded into $35-\mathrm{mm}$ plates and were cultured in DMEM with $10 \%$ fetal calf serum but without antibiotics. When cells had grown to $60-70 \%$ confluence, TJ905 glioma cells were transfected with plasmids of empty pcDNA3.1, pcDNA3.1-anti-hTR and pcDNA3.1-sense-hTR, respectively, using Lipofectamine 2000 (Invitrogen) according to the manufacturer's protocol. Each plate was transfected with a mixture containing $2 \mu \mathrm{g}$ plasmid DNA and $7.5 \mu \mathrm{l}$ liposome. After transfection (5 h), the medium was replaced with fresh DMEM containing serum but not antibiotics, and the cells were incubated for an additional $48 \mathrm{~h}$.

Cell survival assay. The effect of antisense hTR on the growth of human glioma cells was determined by MTT assay using the CellTiter 96 Aqueous One solution cell proliferation assay kit (Promega) according to the manufacturer's instructions. Absorbance (A) readings were taken using a 96-well Opsys MR $^{\mathrm{TM}}$ microplate reader (ThermoLab Systems, Chantily, VA, USA) and Revelation ${ }^{\text {TM }}$ QuickLink software at $490 \mathrm{~nm}$. The blank control wells were used for zeroing absorbance. The percentage of cell survival was calculated using backgroundcorrected absorbance as follows: $\%$ cell viability $=100 \mathrm{x}$ (1-A of experimental well)/A of untreated control well. All experiments were performed at least three times and representative data is presented.

Telomerase activity assay. Telomerase activity was measured by the polymerase chain reaction (PCR)-based telomeric repeats amplification protocol (TRAP) assay (9) using the TRAPeze kit (Intergen Co., Oxford, UK). In brief, after extension of the substrate $5^{\prime}-\left[{ }^{32} \mathrm{P}\right]$-end-labeled TS primer oligonucleotide (5'-AATCCGTCGAGCACAGAGTT-3') by telomerase, the enzyme activity products were amplified by PCR and resolved in $10 \%$ polyacrylamide gel. Each reaction product was amplified in the presence of a 36-bp internal TRAP assay standard. Protein extracts were also incubated at $85^{\circ} \mathrm{C}$ for $10 \mathrm{~min}$ to test their heat sensitivity. A TSR 8 quantitation standard (which serves as a standard to estimate the amount of product extended by telomerase in a given extract) was included for each set of TRAP assays. Telomeric repeat amplification protocol (TRAP reaction) was as follows: i) primer elongation: transfer $25 \mu 1$ reaction mixture into a tube suitable for PCR amplification, then add $2 \mu 1$ cell extract and sterile water to a final volume of $50 \mu \mathrm{l}$; transfer the tubes to a thermal cycler and perform one cycle at $37^{\circ} \mathrm{C}$ for $20 \mathrm{~min}$; ii) telomerase inactivation: perform one cycle at $85^{\circ} \mathrm{C}$ for $5 \mathrm{~min}$; iii) amplification: $50 \mu 1$ reaction mixture contains dNTP, Taq polymerase, the $\left.{ }^{32} \mathrm{P}\right]$-labeled-TS primer, and RP primer (5'-GCGCGGCTTACCCTTACCCTTACCCTAACC-3'). Perform 30 cycles for amplification, then at $94^{\circ} \mathrm{C}$ for $45 \mathrm{sec}$ for denaturation, at $60^{\circ} \mathrm{C}$ for $45 \mathrm{sec}$ for annealing, at $72^{\circ} \mathrm{C}$ for $45 \mathrm{sec}$ for polymerization, and at $72^{\circ} \mathrm{C}$ for $5 \mathrm{~min}$ for balance. Quantitative analysis was performed using Image-Quant software (Molecular Dynamics, Sunnyvale, CA, USA), which allowed densitometric evaluation of the digitalised image. Telomerase activity was quantified by measuring the signal of telomerase ladder bands, and the relative telomerase activity was calculated as the ratio to the internal standard using the following formula: $\left[\left(\mathrm{X}-\mathrm{X}_{0}\right) / \mathrm{C}\right] \times\left[\left(\mathrm{R}-\mathrm{R}_{0}\right) / \mathrm{Cr}\right]^{-1} \mathrm{x} 100=$ total product generated (TPG), where $\mathrm{X}$ is the heat-untreated sample, $\mathrm{X}_{0}$ is the heat-treated sample, $\mathrm{C}$ is the internal control of heat-untreated samples, $\mathrm{Cr}$ is the internal control of TSR8, $\mathrm{R}$ is the TSR8 quantitation control, and $\mathrm{R}_{0}$ is the negative control. The extract of 293 cell line having telomerase activity was used as a positive control (provided by the kit).

Detection of hTR and hTERT $m R N A$ expression by reverse transcription-polymerase chain reaction $(R T-P C R)$. Total RNA was extracted from oligomer-treated cells using a TRIzol RNA kit (Life Technologies, New York, NY, USA). MMLV reverse transcriptase (Gibco) was used to synthesize cDNA. The primers used for $h T E R T, h T R$ and $\beta$-actin gene amplification are shown in Table I. The conditions for PCR were as follows: hTERT: $94^{\circ} \mathrm{C}, 45 \mathrm{sec} ; 55.3^{\circ} \mathrm{C}, 60 \mathrm{sec}$; and $72^{\circ} \mathrm{C}, 75 \mathrm{sec}$ for 
Table I. RT-PCR primer sequences and amplified products of $h T E R T, h T R$, and $\beta$-actin genes.

\begin{tabular}{llll}
\hline Gene & \multicolumn{1}{c}{ Upstream primer } & Downstream primer & Fragment (bp) \\
\hline hTERT & 5'-AAGTTCCTGCACTGGCTGATGAG-3' & 5'-TCGTAGTTGAGCACGCTGAACAG-3' & 377 \\
$h T R$ & 5'-CGCCTTCCACCGTTCATTC-3' & 5'-ATGTGTGAGCCGAGTCCTG-3' & 308 \\
$\beta$-actin & 5'-GGCGGCACCCACCATGTACCCT-3' & 5'-AGGGGCCGGACTCGTCATACT-3' & 220 \\
\hline
\end{tabular}

Table II. The antitumor effect of antisense human telomerase RNA (hTR) on cell survival in human TJ905 glioma cells in vitro as determined by MTT assay.

\begin{tabular}{ccrrrrr}
\hline Time (days) & TJ905 $(\%)$ & TJ905-Ea $(\%)$ & TJ905-Sa $(\%)$ & TJ905-AS $(\%)$ & F & P-value \\
\hline 1 & $100.00 \pm 0.00$ & $97.35 \pm 3.95$ & $100.50 \pm 8.41$ & $94.45 \pm 3.6$ & 41.698 & $>0.05$ \\
2 & $100.00 \pm 0.00$ & $102.96 \pm 5.05$ & $101.20 \pm 7.05$ & $94.28 \pm 4.42$ & 3.575 & $<0.05$ \\
3 & $100.00 \pm 0.00$ & $100.93 \pm 5.87$ & $100.80 \pm 5.57$ & $82.89 \pm 2.61$ & 23.423 & $<0.001$ \\
4 & $100.00 \pm 0.00$ & $105.47 \pm 2.47$ & $101.86 \pm 3.76$ & $78.76 \pm 4.92$ & 78.529 & $<0.001$ \\
5 & $100.00 \pm 0.00$ & $101.11 \pm 1.72$ & $97.28 \pm 3.69$ & $68.59 \pm 2.89$ & 232.389 & $<0.001$ \\
6 & $100.00 \pm 0.00$ & $101.10 \pm 4.55$ & $97.08 \pm 3.95$ & $62.24 \pm 3.59$ & 28.592 & $<0.001$ \\
7 & $100.00 \pm 0.00$ & $100.23 \pm 2.88$ & $95.97 \pm 3.71$ & $52.74 \pm 1.98$ & 492.247 & $<0.001$ \\
\hline
\end{tabular}

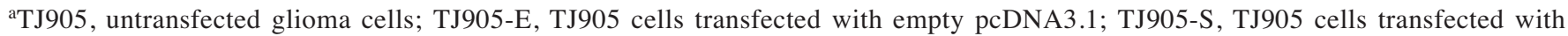
pcDNA3.1-sense-hTR; TJ905-AS, TJ905 cells transfected with pcDNA3.1-anti-hTR. 'P-value, TJ905-AS versus TJ905-E.

31 cycles; hTR: $94^{\circ} \mathrm{C}, 45 \mathrm{sec} ; 53^{\circ} \mathrm{C}, 60 \mathrm{sec}$; and $72^{\circ} \mathrm{C}, 75 \mathrm{sec}$ for 30 cycles; and $\beta$-actin: $94^{\circ} \mathrm{C}, 45 \mathrm{sec} ; 56^{\circ} \mathrm{C}, 60 \mathrm{sec}$; and $72^{\circ} \mathrm{C}, 75 \mathrm{sec}$ for 31 cycles. PCR products were resolved by agarose gel electrophoresis, and levels of bands on gel were measured using a gel-image system.

In vivo tumor model study. A total of $3 \times 10^{7}$ TJ905 glioma cells was transplanted s.c. into NOD/LtSz-Prkdc(scid)/J (NOD/SCID) mice. After the tumor was established, the mice were divided into three groups (12 mice per group) and injected with liposome only (TJ905), empty vector in liposome (TJ905-E) and anti-hTR vector in liposome (TJ905-AS), respectively. Tumor dimensions were measured with calipers every three days. Mean tumor size was calculated as width ${ }^{2} \mathrm{x}$ length x 0.52 (10). All measurements were performed in a coded, blinded fashion. Mice were sacrificed 33 days later, and tumors were resected to determine telomerase activity by TRAP assay and to detect the expression of hTR and hTERT mRNA by RT-PCR analysis.

Statistical analysis of data. Data were analyzed with SPSS 10.0 , followed by variance analysis for comparison among different groups. A $\mathrm{P}<0.05$ was considered statistically significant.

\section{Results}

The effect of antisense hTR cDNA on cell proliferation in TJ905 human glioma cells in vitro. To understand the antitumor effects of antisense hTR in human cancers, we first established whether antisense hTR cDNA inhibits glioma cell proliferation. The human glioma cell line, TJ905, was used as a model for human glioma because its telomerase activity is very high. The ability of antisense hTR to inhibit cellular growth was investigated in vitro using the MTT-based colorimetric assay. As shown in Fig. 1 and Table II, antisense hTR cDNA caused a marked inhibition of TJ905 cell proliferation in a timedependent manner in vitro. Starting from the second day, the survival rate of TJ905 cells transfected with antisense hTR cDNA began to decrease, and it reached $52.7 \%$ compared to untransfected cells or cells transfected with the empty plasmid, pcDNA3.1, on the seventh day. In contrast, the survival rate of TJ905 cells transfected with sense hTR cDNA was $95.97 \%$ on the seventh day as compared to the controls (Table II and Fig. 1).

The effect of antisense hTR cDNA on cell growth in TJ905 human glioma cells in vivo. The tumors of the TJ905-AS group grew very slowly in vivo and their dimensions were considerably smaller than those in the control groups (TJ905 and TJ905-E). There was a significant difference in tumor size between the TJ905-AS group and the control groups, even on the third day of the study, and there existed striking differences in tumor mass between the TJ905-AS group and the control groups at the termination of the study $(\mathrm{P}<0.001)$. As seen in Table III and Fig. 2, the average glioma volume in the TJ905-AS group on day 33 of the study was only $446.1 \mathrm{~mm}^{3}$. In contrast, the average sizes of the tumor in the TJ905 and TJ905-S groups were 2914.36 and $2834.76 \mathrm{~mm}^{3}$, respectively (Table III and Fig. 2). 


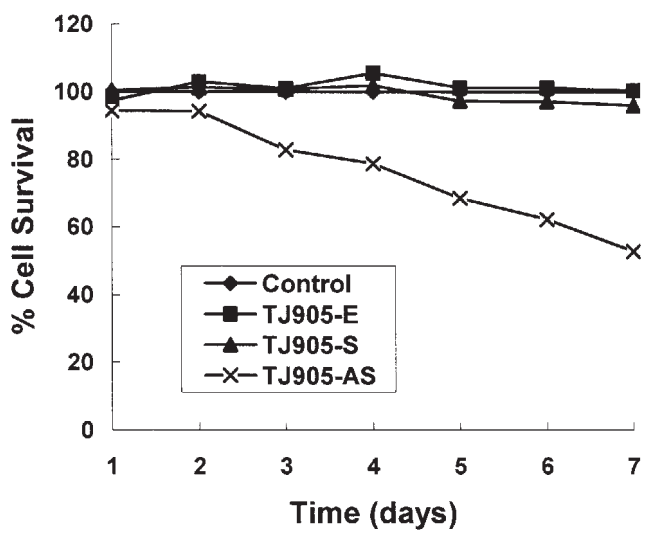

Figure 1. Inhibitory effect of antisense human telomerase RNA (hTR) on cell proliferation in human TJ905 glioma cells in vitro. TJ905 cells were transfected with empty pcDNA3.1 (TJ905-E), pcDNA3.1-sense-hTR (TJ905-S) and pcDNA3.1-anti-hTR (TJ905-AS), respectively, or left untransfected as a control (TJ905), and cell survival was assessed by MTT assay as described in Materials and methods. Cell growth values were expressed relative to those of TJ905-E cells (100\% control value). The results represent the mean of at least three independent experiments.

The levels of hTR and hTERT were significantly reduced in vitro and in vivo in TJ905 glioma cells treated with antisense hTR cDNA. Because antisense hTR effectively inhibited human glioma cell growth, we determined the levels of hTR and hTERT in the tumor cells in vitro and in vivo. We transfected the TJ905 glioma cells with pcDNA3.1, pcDNA3.1-anti-hTR and pcDNA3.1-sense-hTR, respectively, and determined the levels of hTR and hTERT mRNA expression in these cells by RT-PCR analysis. As can be observed in Table IV and Figs. 3 and 4, the levels of hTR and hTERT mRNA were considerably lower in TJ905 cells transfected with antisense hTR cDNA than in untransfected tumor cells and cells

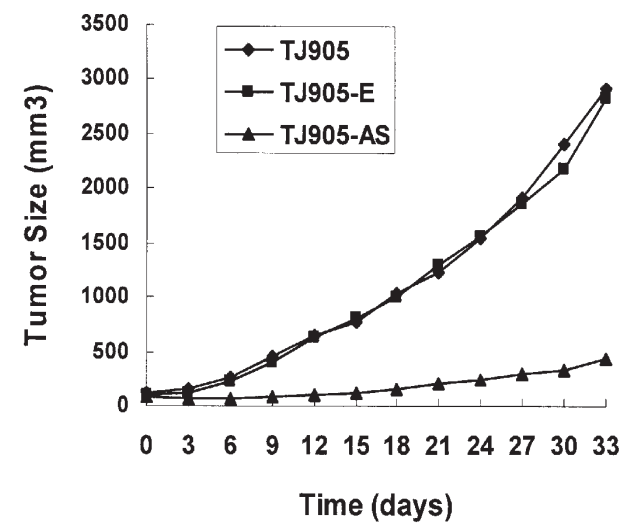

Figure 2. Anticancer effect of antisense human telomerase RNA (hTR) on the growth of human TJ905 glioma in nude mice. A total of $3 \times 10^{7}$ TJ905 glioma cells was transplanted s.c. into NOD/SCID mice. After tumors were established in mice, the mice were placed in three groups and injected with liposome only (TJ905), empty vector in liposome (TJ905-E) and anti-hTR vector in liposome (TJ905-AS), respectively. The ability of antisense hTR cDNA to inhibit tumor growth in vivo was determined by measuring the tumor volume, as described in Materials and methods. Tumor dimensions were measured using calipers every three days. Mean tumor size was calculated as width ${ }^{2} \mathrm{x}$ length $\mathrm{x} 0.52$. All measurements were performed in a coded, blinded fashion.

transfected with empty vector or sense hTR cDNA. Similar data were also obtained from our in vivo animal model study. After TJ905 gliomas were established in the nude mice, the tumors were injected with antisense hTR vector (TJ905-AS), or with liposome only (TJ905) or the empty plasmid (TJ905-E) as controls. We found that the levels of hTR and hTERT mRNA expression were significantly decreased in cells injected with anti-hTR cDNA compared to cells in the control groups, as analyzed by RT-PCR (Table IV and Figs. 3 and 4).

Table III. The inhibitory effect of antisense human telomerase RNA (hTR) on the growth of TJ905 human gliomas in vivo as determined by measuring tumor volume.

\begin{tabular}{|c|c|c|c|c|c|}
\hline Time (days) & TJ905 $\left(\mathrm{mm}^{3}\right)$ & TJ905-Ea $\left(\mathrm{mm}^{3}\right)$ & TJ905-AS $\left(\mathrm{mm}^{3}\right)$ & $\mathrm{F}$ & P-value \\
\hline 0 & $115.74 \pm 27.87$ & $96.88 \pm 9.96$ & $88.03 \pm 24.54$ & 2.524 & $>0.05$ \\
\hline 3 & $158.04 \pm 28.74$ & $131.48 \pm 6.63$ & $73.14 \pm 23.01$ & 22.354 & $<0.001$ \\
\hline 6 & $264.71 \pm 27.00$ & $229.79 \pm 35.86$ & $70.38 \pm 24.98$ & 62.356 & $<0.001$ \\
\hline 9 & $455.03 \pm 80.30$ & $411.00 \pm 106.92$ & $87.94 \pm 24.25$ & 31.392 & $<0.001$ \\
\hline 12 & $642.14 \pm 105.24$ & $629.59 \pm 162.29$ & $101.72 \pm 32.99$ & 35.436 & $<0.001$ \\
\hline 15 & $775.99 \pm 98.58$ & $802.35 \pm 207.38$ & $116.00 \pm 48.47$ & 39.006 & $<0.001$ \\
\hline 18 & $1040.73 \pm 223.48$ & $1003.40 \pm 254.59$ & $155.20 \pm 70.32$ & 30.861 & $<0.001$ \\
\hline 21 & $1238.17 \pm 144.62$ & $1303.91 \pm 331.90$ & $211.80 \pm 87.70$ & 38.476 & $<0.001$ \\
\hline 24 & $1553.70 \pm 160.12$ & $1567.28 \pm 463.93$ & $251.60 \pm 103.38$ & 31.758 & $<0.001$ \\
\hline 27 & $1915.82 \pm 173.89$ & $1861.44 \pm 521.23$ & $304.40 \pm 117.34$ & 36.957 & $<0.001$ \\
\hline 30 & $2414.66 \pm 306.58$ & $2176.88 \pm 651.08$ & $334.30 \pm 112.70$ & 34.136 & $<0.001$ \\
\hline 33 & $2914.36 \pm 341.83$ & $2836.76 \pm 692.92$ & $446.10 \pm 196.28$ & 44.100 & $<0.001$ \\
\hline
\end{tabular}

${ }^{a}$ TJ905, TJ905 glioma injected with liposome only; TJ905-E, TJ905 glioma injected with empty pcDNA3.1; TJ905-AS, TJ905 glioma

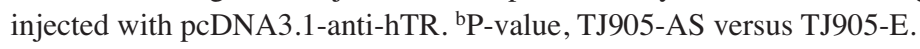


Table IV. The inhibitory effect of antisense human telomerase RNA (hTR) on the levels of hTR and hTERT mRNA expression in human TJ905 glioma cells in vitro and in vivo as assessed by RT-PCR analysis.

\begin{tabular}{|c|c|c|c|c|}
\hline \multirow[b]{2}{*}{ Group } & \multicolumn{2}{|c|}{ Level of hTR } & \multicolumn{2}{|c|}{ Level of hTERT } \\
\hline & In vitro & In vivo & In vitro & In vivo \\
\hline TJ905 & $1.581 \pm 0.016^{\mathrm{b}}$ & $1.495 \pm 0.045^{\mathrm{b}}$ & $0.496 \pm 0.051^{\mathrm{b}}$ & $0.509 \pm 0.063^{b}$ \\
\hline TJ905-E & $1.511 \pm 0.057^{\mathrm{b}}$ & $1.483 \pm 0.127^{\mathrm{b}}$ & $0.494 \pm 0.068^{\mathrm{b}}$ & $0.459 \pm 0.084^{b}$ \\
\hline TJ905-S & $1.559 \pm 0.168^{\mathrm{b}}$ & & $0.487 \pm 0.041^{\mathrm{b}}$ & \\
\hline TJ905-AS & $0.356 \pm 0.041$ & $0.418 \pm 0.051$ & $0.022 \pm 0.004$ & $0.023 \pm 0.042$ \\
\hline $\mathrm{F}$ & 121.324 & 263.799 & 122.986 & 92.790 \\
\hline P-value & $<0.001$ & $<0.001$ & $<0.001$ & $<0.001$ \\
\hline
\end{tabular}

aTJ905, untransfected or untreated glioma cells; TJ905-E, TJ905 cells transfected or injected with empty pcDNA3.1; TJ905-S, TJ905 cells transfected with pcDNA3.1-sense-hTR; TJ905-AS, TJ905 cells transfected or injected with pcDNA3.1-anti-hTR. ${ }^{\text {bP }}<0.05$ versus TJ905-AS.

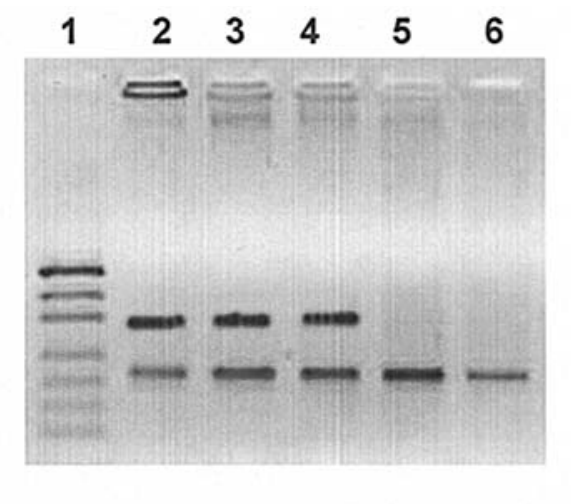

In vitro

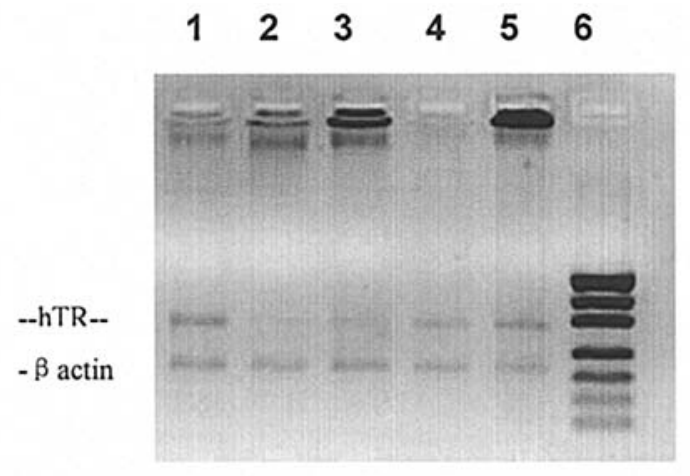

In vivo

Figure 3. Reverse transcription-polymerase chain reaction (RT-PCR) analysis of the levels of hTR mRNA expression in human glioma cells in vitro and in vivo. TJ905 cells were transfected in vitro or injected in vivo with empty pcDNA3.1 (TJ905-E), pcDNA3.1-sense-hTR (TJ905-S) and pcDNA3.1-anti-hTR (TJ905-AS), respectively, or left untransfected or untreated as controls (TJ905). Total cellular RNA was isolated from glioma cells using TRIzol reagent, and the mRNA expression of $h T R$ and $\beta$-actin genes was analyzed by RT-PCR. Numeric data are shown in Table IV. In vitro: lane 1 , marker; lane 2 , TJ905; lane 3 , TJ905-S; lane 4, TJ905-E; lanes 5 and 6, TJ905-AS. In vivo: lane 1, TJ905; lanes 2 and 3, TJ905-AS; lane 4, TJ905-S; lane 5, TJ905-E; lane 6, marker.

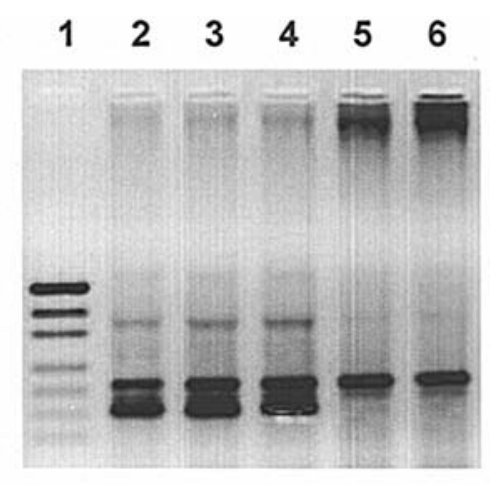

In vitro

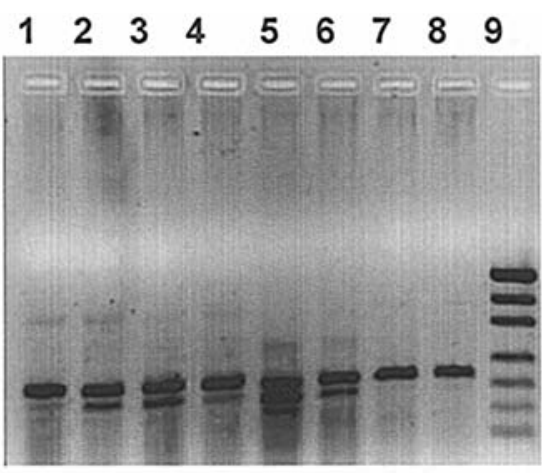

In vivo

Figure 4. Detection of hTERT mRNA expression in human glioma cells in vitro and in vivo by reverse transcriptase-PCR. TJ905 cells were transfected in vitro or injected in vivo with empty pcDNA3.1 (TJ905-E), pcDNA3.1-sense-hTR (TJ905-S) and pcDNA3.1-anti-hTR (TJ905-AS), respectively, or left untransfected or untreated as controls (TJ905). Total cellular RNA was isolated from glioma cells using TRIzol reagent, and the mRNA expression of $h T E R T$ and $\beta$-actin genes was analyzed by RT-PCR. Numeric data are shown in Table IV. In vitro: lane 1, marker; lane 2, TJ905; lane 3, TJ905-S; lane 4, TJ905-E; lanes 5 and 6, TJ905-AS. In vivo: lane 1, TJ905; lane 2, TJ905-E; lanes 3 and 4, TJ905-S; lanes 5-8, TJ905-AS; lane 9, marker. 


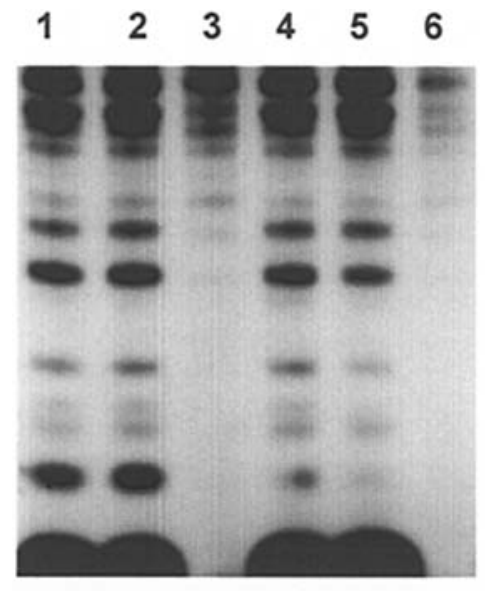

In vitro

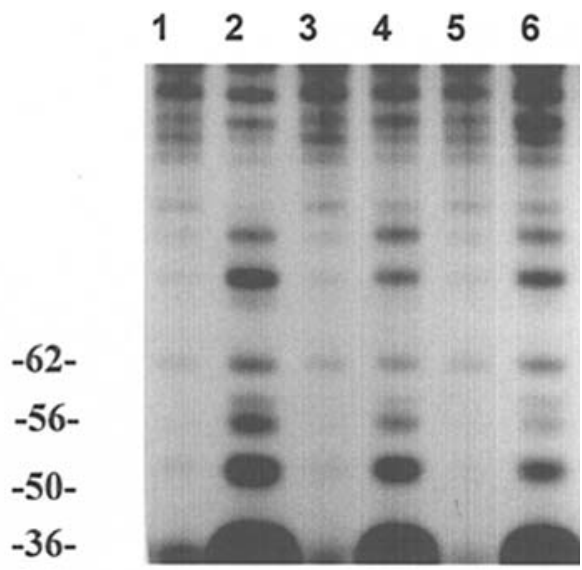

In vivo

Figure 5. Assessment of the effect of antisense human telomerase RNA (hTR) on telomerase activity in human glioma cells in vitro and in vivo by TRAP assay. TJ905 cells were transfected in vitro or injected in vivo with empty pcDNA3.1 (TJ905-E), pcDNA3.1-sense-hTR (TJ905-S) and pcDNA3.1-anti-hTR (TJ905-AS), respectively, or left untransfected or untreated as controls (TJ905). Telomerase activity was measured by TRAP assay, as described in Materials and methods. Numeric data are shown in Table V. In vitro: lane 1, TJ905-E; lane 2, TJ905; lanes 3 and 6, TJ905-AS; lanes 4 and 5, TJ905-S. In vivo: lanes 1, 3, and 5, TJ905-AS; lanes 2 and 4, TJ905; lane 6, TJ905-E.

Table V. The inhibitory effect of antisense human telomerase RNA (hTR) on telomerase activity in human TJ905 glioma cells in vitro and in vivo as measured by TRAP assay.

\begin{tabular}{lcc}
\hline \multirow{2}{*}{ Group } & \multicolumn{2}{c}{ Telomerase activity (TPG) } \\
\cline { 2 - 3 } & In vitro & In vivo \\
\hline TJ905 $^{\mathrm{a}}$ & $332.2 \pm 22.1^{\mathrm{b}}$ & $441.2 \pm 51.6^{\mathrm{b}}$ \\
TJ905-E $^{\mathrm{a}}$ & $348.2 \pm 44.9^{\mathrm{b}}$ & $436.0 \pm 52.2^{\mathrm{b}}$ \\
TJ905-S $^{\mathrm{a}}$ & $336.2 \pm 18.0^{\mathrm{b}}$ & \\
TJ905-AS $^{\mathrm{a}}$ & $49.0 \pm 13.3$ & $49.8 \pm 17.1$ \\
$\mathrm{~F}$ & 131.63 & 132.67 \\
$\mathrm{P}$ & $<0.001$ & $<0.001$ \\
\hline
\end{tabular}

${ }^{a}$ TJ905, untransfected or untreated glioma cells; TJ905-E, TJ905 cells transfected or injected with empty pcDNA3.1; TJ905-S, TJ905 cells transfected with pcDNA3.1-sense-hTR; TJ905-AS, TJ905 cells transfected or injected with pcDNA3.1-anti-hTR. ${ }^{\text {b }} \mathrm{P}<0.05$ versus TJ905-AS.

The effect of antisense hTR on telomerase activity in TJ905 glioma cells in vitro and in vivo. As we found that antisense hTR could inhibit human TJ905 glioma cell proliferation and tumor growth in vitro and in vivo, we reasoned that this inhibitory activity may be attributable to its ability to interfere with telomerase activity. We therefore analyzed the telomerase activity in glioma cells in which the expression of hTR and hTERT mRNA was suppressed by antisense hTR. TJ905 glioma cell transfection was performed using vectors or the in vivo tumor model was injected with the plasmid constructs, as described in Materials and methods, and the telomerase activity in glioma cells from both in vitro and in vivo studies was measured by TRAP method. Our results revealed that the telomerase activity in TJ905 cells was dramatically decreased by antisense hTR cDNA compared to the control cells. As seen in Table V and Fig. 5, the telomerase activity in the TJ905-AS group was only 49.0 TPG in vitro and $49.8 \mathrm{TPG}$ in vivo. In contrast, the telomerase activity ranged from 332.2 TPG to $348.2 \mathrm{TPG}$ in vitro and from 436.0 TPG to 441.2 TPG in vivo in the control groups. These data suggest that the inhibitory effect of antisense hTR on the growth of human glioma cells in vitro and in vivo may be through downregulating hTR and hTERT expression and suppressing telomerase activity.

\section{Discussion}

Telomerase, a ribonucleoprotein enzyme that functions as a reverse transcriptase, is detected exclusively in immortal cells, such as germ cells, stem cells, and cancer cells. Telomerase activity is present in almost all human cancers. It is well known that telomerase activation and subsequent maintenance of telomeres are essential to maintain the integrity of the replicating tumor cell and establish immortality and, thus, are required for the survival and proliferation of the large majority of tumor cells. Therefore, strategies targeting telomerase may be a new approach for gene therapy of cancer. Because hTR provides the template for the de novo synthesis of telomeric DNA and overcomes the 'end-replication problem', we selected hTR, including its template, as the target of antisense gene therapy and investigated the influence of antisense hTR cDNA on the growth of TJ905 glioma cells. We have demonstrated that cell growth in TJ905 glioma was inhibited significantly in vitro and in vivo following transfection or injection with antisense hTR cDNA. In addition, we found that the levels of hTR mRNA expression and telomerase activity in these cells were substantially decreased. These observations suggest that the antiproliferative effect and antineoplastic activity of antisense hTR in human glioma cells are mediated through 
suppression of telomerase activity. These findings also provide the rationale for the development of antisense hTR as a potentially effective gene therapy for patients with malignant gliomas and other brain tumors.

In recent years, many different antisense gene therapies have been reported to achieve telomerase inhibition in tumor cells. Several experimental studies, mainly based on the use of antisense oligonucleotides targeting hTR, showed that telomerase inhibition was maintained for a long time and resulted in tumor cell growth arrest. Targeting hTR resulted in delayed tumor cell growth as a consequence of progressive telomere shortening when telomerase is inhibited. In fact, as human cells reduce their telomere length by 50-200 bp per cell division, a long lag phase is required before growth arrest can be obtained, even in tumor cells with relatively short telomeres.

Human telomeres are DNA protein structures consisting of tanden repetitive hexameric sequences (TTAGGG) $)_{n}$ in mammals, located at the end of linear chromosomes and composed of multiple copies of short, repetitive sequences of DNA spanning 5-15 kb. They have an important protective function in preventing chromosomes from undergoing aberrant recombination and end-to-end fusion and are involved in the senescence process, acting as a molecular clock that controls the life-span of human cells (11-13). In most somatic cells, telomeres are progressively shortened by $50-200$ bp per cell division during each replication cycle, as a result of incomplete DNA replication at the lagging strand, the so-called 'endreplication problem'. Immortal cells and cancer cells acquire immortalized growth capacity by stabilizing the length of their telomeres through the reactivation of telomerase, a unique reverse transcriptase that synthesizes telomeric repeats. Hence, the 'end-replication problem' is overcome in cancer cells through this mechanism. Telomerase expression has been detected in more than $90 \%$ of malignant tumors, while only a small fraction of cancers maintain telomeres through an alternative lengthening of telomere recombination mechanism, but it is absent in the great majority of normal somatic tissues, with a few exceptions including germ-line cells, embryonic stem cells, activated lymphocytes, and cells from the basal layer of the epidermis. The higher the degree of malignancy of the tumor, the higher the level of telomerase activity. The telomerase core enzyme consists of an RNA component (hTR) that provides the template for the de novo synthesis of telomeric DNA and hTERT. It has been shown that hTR is expressed in all human tissues regardless of telomerase activity (14). hTR was cloned in 1995 by Feng et al (15). While hTERT is the catalytic subunit of telomerase with reverse transcriptase activity, hTERT expression can be detected in the majority of tumors but is absent in most normal somatic tissue (16). The expression of the hTERT component is closely associated with the enzyme catalytic activity in telomerase-positive cells and, therefore, is the primary determinant for the activity of telomerase. hTERT expression is usually parallel to telomerase activity (17-23). The relatively tumor-restricted expression of telomerase has made the enzyme an important target for the development of new anticancer therapies.

The application of antisense gene therapy can inhibit the template effect of hTR and telomerase activity and prevent cellular immortality. Kim et al (1) found that antisense hTR oligonucleotides could prevent telomerase from synthesizing telomeric repeats. Feng and co-workers (15) used antisense gene therapy against the template of hTR. They added antisense hTR against the template of hTR into the culture medium of tumor cells and found that tumor cells entered a growth crisis after a period of time, accompanied by shortening of the telomere and inhibition of telomerase activity. Kondo and colleagues $(6,7)$ treated human malignant glioma cells with antisense hTR oligonucleotides and showed that antisense gene therapy not only inhibited telomerase activity but also inhibited the growth of tumor cells in vivo and in vitro, reduced the volume of tumors, decreased the number of metastatic nodes and induced differentiation of tumor cells.

In the present study, we constructed an expression vector consisting of hTR antisense cDNA against the template of hTR and examined its effect on human TJ905 glioma cells in vitro. We observed a remarkable reduction of cell growth within a few days of the treatment. Starting from the second day, the cell survival rate began to decrease in a time-dependent manner, and the survival rate had reduced to $52.7 \%$ on the seventh day. This decline in cell survival appears to be a direct consequence of hTR antisense cDNA-mediated interference with hTR expression. Meanwhile, the telomerase activity of the cells was inhibited, and the levels of hTR and hTERT mRNA expression were reduced significantly. The telomerase activity in the treatment groups was 49.0 TPG, which was remarkably decreased compared with that of the control groups, and the levels of hTR and hTERT mRNA in the treatment groups were 0.356 and 0.022 , respectively, which were substantially lower than those in the control groups (Table IV). Similar results were obtained from our in vivo study. We demonstrated that the growth of glioma in nude mice was inhibited significantly after transfection with hTR antisense cDNA, that the telomerase activity in the tumor was decreased and the levels of hTR and hTERT mRNA expression were reduced significantly. Our results have potential relevance for a better understanding of the effect of interference with hTR on the proliferative potential of tumor cells. The possible mechanism involved may be that some of the hTR in the glioma cells was destroyed by the hTR antisense cDNA and caused a reduction in telomerase activity leading to the growth inhibition of tumor cells. Interestingly, we also found that the level of hTERT mRNA expression was decreased after transfection or injection with hTR antisense cDNA. However, the underlying mechanism for this reduction remains to be experimentally elucidated.

In conclusion, we have assessed the effect of antisense hTR cDNA on cell growth in glioma cells and found that it effectively inhibited TJ905 human glioma cell proliferation and tumor growth in vitro and in vivo. We also demonstrated that telomerase activity and the levels of hTR and hTERT mRNA expression were markedly reduced in these cells after they were treated with antisense hTR cDNA, suggesting that the inhibitory effect of antisense hTR on glioma cell growth is through repression of hTR and hTERT expression and suppression of telomerase activity. These data support the potential therapeutic applications of antisense hTR in malignant gliomas in the clinic. Finally, as mentioned above, we also observed that the level of hTERT mRNA expression decreased 
following treatment with hTR antisense cDNA, and hTERT has been shown to play an important part in regulating the telomerase activity in different tumors. Therefore, further research is necessary to explore the potential of hTERT as a novel target of gene therapy for human gliomas (8) and other malignancies.

\section{Acknowledgments}

This publication was made possible by a National Institutes of Health grant (no. P20RR16440-010003 to Q.Q.L.).

\section{References}

1. Kim NW, Piatysrek MA and Prowse KR: Specific association of human telomerase activity with immortal cells and cancer. Science 266: 2011-2015, 1994.

2. You Y, Pu P and Peng Q: Telomerase activity and regulation in human neuroepithelial tumors. Chin J Surg 40: 90-93, 2002.

3. Shay JW and Bacchetti S: A survey of telomerase activity in human cancer. Eur J Cancer 33: 787-791, 1997.

4. Harada K, Arita K and Kurisu K: Telomerase activity and the expression of telomerase components in pituitary adenoma with malignant transformation. Surg Neurol 53: 267-274, 2000.

5. Hiraga S: Telomerase activity and alterations in telomere length in human brain tumors. Cancer Res 58: 2117-2125, 1998.

6. Kondo Y, Koga S and Komata T: Treatment of prostate cancer in vitro and in vivo with 2-5A-anti-telomerase RNA component. Oncogene 19: 2205-2211, 2000.

7. Mukai S, Kondo Y and Koga S: 2-5A antisense telomerase RNA therapy for intracranial malignant gliomas. Cancer Res 60: 4461-4467, 2000.

8. Komata T, Kondo Y and Kanzawa T: Treatment of malignant glioma cells with the transfer of constitutively active caspase-6 using the human telomerase catalytic subunit (human telomerase reverse transcriptase) gene promoter. Cancer Res 61: 5796-5802, 2001.

9. Kim NM and Wu F: Advances in quantification and characterization of telomerase activity by the telomeric repeat amplification protocol (TRAP). Nucleic Acids Res 25: 2595-2597, 1997.
10. Hahnfeldt P, Panigrahy D, Folkman J and Hlatky L: Tumor development under angiogenic signaling: a dynamical theory of tumor growth, treatment response, and postvascular dormancy. Cancer Res 59: 4770-4775, 1999.

11. Nurnberg P, Thiel G and Weber F: Changes of telomere lengths in human intracranial tumors. Hum Genet 91: 190-192, 1993.

12. De Lange T: Telomeres and senescence: ending the debate. Science 279: 334-335, 1998 .

13. Harley CB, Futcher AB and Greider CW: Telomeres shorten during age of human fibroblasts. Nature 345: 458-460, 1990.

14. Ariel A, Avilion L and Mieczyslaw A: Human telomerase RNA and telomerase activity in immortal cell lines and tumor tissues. Cancer Res 56: 645-650, 1996.

15. Feng J, Funk WD and Sy SW: The RNA component of human telomerase. Science 269: 1236-1241, 1995.

16. Meyerson M, Counter CM and Eaton EN: hEST2, the putative human telomerase catalytic subunit gene, is up-regulated in tumor cells and during immortalization. Cell 90: 785-795, 1997.

17. Suzuki T, Suzuki Y and Fujioka T: Expression of the catalytic subunit associated with telomerase gene in human urinary bladder cancer. J Urol 162: 2217-2220, 1999.

18. Kyo S, Kanaya T and Takakura M: Human telomerase reverse transcriptase as a critical determinant of telomerase activity in normal and malignant endometrial tissues. Int J Cancer 80: 60-63, 1999

19. Harrington L, McPhail T and Mar V: A mammalian telomeraseassociated protein. Science 275: 973-977, 1997.

20. Dome JS, Chung S and Bergemann T: High telomerase reverse transcriptase (hTERT) messenger RNA level correlates with tumor recurrence in patients with favorable histology Wilm's tumor. Cancer Res 59: 4301-4307, 1999.

21. Hisatomi H, Nagao K and Kanamaru T: Levels of telomerase catalytic subunit mRNA as a predictor of potential malignancy. Int J Oncol 14: 717-732, 1999.

22. Simon M, Park TW and Leuenroth S: Telomerase activity and expression of the telomerase catalytic subunit, hTERT, in meningioma progression. J Neurosurg 92: 832-840, 2000.

23. Beattie TL, Zhou W and Robinson MO: Reconstitution of human telomerase activity in vitro. Curr Biol 8: 177-180, 1998. 